\title{
Electric quadrupole transitions of the Bohr Hamiltonian with the Morse potential
}

\author{
I. Inci ${ }^{1,3}$, D. Bonatsos ${ }^{2}$, and I. Boztosun ${ }^{3}$ \\ 1 I.N.F.N. and Dipartimento di Fisica "Galileo Galilei", \\ Universitá di Padova, I-35131, Padova, Italy \\ 2 Institute of Nuclear Physics, National Centre for Scientific Research "Demokritos", \\ GR-15310 Aghia Paraskevi, Attiki, Greece and \\ 3 Department of Physics, Akdeniz University, TR-07058, Antalya, Turkey
}

\begin{abstract}
Eigenfunctions of the collective Bohr Hamiltonian with the Morse potential have been obtained by using the Asymptotic Iteration Method (AIM) for both $\gamma$-unstable and rotational structures. $B(E 2)$ transition rates have been calculated and compared to experimental data. Overall good agreement is obtained for transitions within the ground state band, while some interband transitions appear to be systematically underpredicted in $\gamma$-unstable nuclei and overpredicted in rotational nuclei.
\end{abstract}

PACS numbers: 21.30.Fe, 21.60.Ev, 21.60.Fw, 21.10.Re

Keywords: Bohr Hamiltonian, Morse potential, electric quadrupole transitions

\section{INTRODUCTION}

Shape phase transitions in atomic nuclei have attracted much attention in the last decade [1], following the introduction of the critical point symmetries $\mathrm{E}(5)$ [2] and $\mathrm{X}(5)$ [3]. $\mathrm{E}(5)$ describes the second-order phase transition point between vibrational and $\gamma$-unstable nuclei, the parameter-free (up to overall scale factors) properties of such a structure obtained by using an infinite-well potential in the collective Bohr Hamiltonian [4]. The first-order phase transition point between vibrational and axially symmetric prolate deformed rotational nuclei is given by $\mathrm{X}(5)$, the structural properties again being found from a solution of the Bohr Hamiltonian with an infinite-well potential [3] .

One way to describe nuclei which are close to or away from these critical points is getting special solutions of the Bohr Hamiltonian with a suitable potential. By using Coulomblike and Kratzer-like [5] potentials in the Bohr Hamiltonian, eigenvalues and eigenfunctions have been obtained in closed forms for the $\gamma$-unstable region [6]. In the deformed rotational region, analytical solutions of the Bohr Hamiltonian using a Coulomb-like or Kratzer-like potential for the $\beta$-part of the potential and a harmonic oscillator potential for the $\gamma$ part of the potential have been found by assuming that the Hamiltonian is separable into its variables [7]. For both regions, $\beta^{2 n}$-type $(n=1,2,3,4)$ [8 10] and Davidson [11 15] potentials have been used in the Bohr Hamiltonian and spectra and $B(E 2)$ transition rates have been calculated. 
The Morse potential has been used recently [16] in order to solve the over-prediction of the energy spacing problem within the $\beta$-band of X(5) and related solutions [17-19]. Closed expressions for the energy eigenvalues have been obtained using the asymptotic iteration method (AIM) 20, 21]. To complete this project, in the present work the eigenfunctions are constructed and $B(E 2)$ transition rates are calculated and compared to the experimental data in the $\gamma$-unstable and rotational regions, as well as to theoretical predictions of other models.

The paper has the following structure. In Sec. II the asymptotic iteration method (AIM) is briefly described. In Sec. III the wave functions are constructed and the electric quadrupole transition strengths are calculated in the $\gamma$-unstable and rotational regions. Numerical results are given in Sec. IV, while in Sec. V the conclusions and outlook are discussed. Details of the calculations are given in Appendix A.

\section{OVERVIEW OF THE ASYMPTOTIC ITERATION METHOD}

The Asymptotic Iteration Method (AIM) has been proposed [20, 21] and applied [22 28] to the solution of second-order differential equations of the form

$$
y^{\prime \prime}=\lambda_{0}(x) y^{\prime}+s_{0}(x) y,
$$

where $\lambda_{0}(x) \neq 0$ and the prime denotes the derivative with respect to $x$. The functions $s_{0}(x)$ and $\lambda_{0}(x)$ must be sufficiently differentiable. Eq. (10) has a general solution [20]

$$
y(x)=\exp \left(-\int^{x} \alpha\left(x_{1}\right) d x_{1}\right)\left[C_{2}+C_{1} \int^{x} \exp \left(\int^{x_{1}}\left[\lambda_{0}\left(x_{2}\right)+2 \alpha\left(x_{2}\right)\right] d x_{2}\right) d x_{1}\right]
$$

for sufficiently large $k, k>0$, if

$$
\frac{s_{k}(x)}{\lambda_{k}(x)}=\frac{s_{k-1}(x)}{\lambda_{k-1}(x)}=\alpha(x),
$$

where

$$
\begin{aligned}
\lambda_{k}(x) & =\lambda_{k-1}^{\prime}(x)+s_{k-1}(x)+\lambda_{0}(x) \lambda_{k-1}(x), \\
s_{k}(x) & =s_{k-1}^{\prime}(x)+s_{0}(x) \lambda_{k-1}(x), \quad k=1,2,3, \ldots
\end{aligned}
$$

For a given potential, the radial Schrödinger equation is converted to the form of Eq. (11). Then, $\mathrm{s}_{0}(x)$ and $\lambda_{0}(x)$ are determined, and the functions $\mathrm{s}_{k}(x)$ and $\lambda_{k}(x)$ are calculated by the recurrence relations of Eq. (4).

The termination condition of the method, given in Eq. (3), can be arranged as

$$
\Delta_{k}(x)=\lambda_{k}(x) s_{k-1}(x)-\lambda_{k-1}(x) s_{k}(x)=0, \quad k=1,2,3, \ldots
$$

Then, the energy eigenvalues are obtained from the roots of Eq. (5) if the problem is exactly solvable. If not, for a specific principal quantum number $n$, we choose a suitable $x_{0}$ point, generally determined as the maximum value of the asymptotic wave function or the minimum value of the potential [20, 25], and the approximate energy eigenvalues are obtained from the roots of this equation for sufficiently large values of $k$ by iteration. 
The corresponding eigenfunctions can be derived from the following wave function generator for exactly solvable potentials

$$
y_{n}(x)=C_{2} \exp \left(-\int^{x} \frac{s_{n}\left(x_{1}\right)}{\lambda_{n}\left(x_{1}\right)} d x_{1}\right)
$$

where $n$ represents the radial quantum number.

Recently, Boztosun and Karakoc [29] have further improved the method for the exactly solvable problems. This improved form had been applied to the exactly separable $\gamma \approx 0$ solution of the Morse potential in Ref. [16].

\section{B(E2) TRANSITION STRENGTHS}

\section{A. The $\gamma$-unstable case}

The Bohr Hamiltonian [4] is

$$
H=-\frac{\hbar^{2}}{2 B}\left[\frac{1}{\beta^{4}} \frac{\partial}{\partial \beta} \beta^{4} \frac{\partial}{\partial \beta}+\frac{1}{\beta^{2} \sin 3 \gamma} \frac{\partial}{\partial \gamma} \sin 3 \gamma \frac{\partial}{\partial \gamma}-\frac{1}{4 \beta^{2}} \sum_{k=1}^{3} \frac{\hat{Q}_{k}^{2}}{\sin ^{2}\left(\gamma-\frac{2 \pi}{3} k\right)}\right]+V(\beta, \gamma),
$$

where $\beta$ and $\gamma$ are collective coordinates describing the deformation and the shape of a nucleus, while $\hat{Q}_{k}$ represents the angular momentum component in the body-fixed coordinate system and $B$ is the mass parameter. For $\gamma$-unstable structure, the potential energy is independent of $\gamma$, namely $V(\beta, \gamma)=V(\beta)$, and by assuming a wave function of the form [30]

$$
\psi\left(\beta, \gamma, \theta_{i}\right)=\xi(\beta) \Phi\left(\gamma, \theta_{i}\right),
$$

where $\theta_{i}(i=1,2,3)$ are the Euler angles, one can achieve separation of variables

$$
\begin{gathered}
{\left[-\frac{1}{\sin 3 \gamma} \frac{\partial}{\partial \gamma} \sin 3 \gamma \frac{\partial}{\partial \gamma}+\frac{1}{4} \sum_{k=1}^{3} \frac{\hat{Q}_{k}^{2}}{\sin ^{2}\left(\gamma-\frac{2 \pi}{3} k\right)}\right] \Phi\left(\gamma, \theta_{i}\right)=\tau(\tau+3) \Phi\left(\gamma, \theta_{i}\right),} \\
{\left[-\frac{1}{\beta^{4}} \frac{\partial}{\partial \beta} \beta^{4} \frac{\partial}{\partial \beta}+\frac{\tau(\tau+3)}{\beta^{2}}+u(\beta)\right] \xi(\beta)=\epsilon \xi(\beta),}
\end{gathered}
$$

where $\epsilon=\frac{2 B}{\hbar^{2}} E$ and $u(\beta)=\frac{2 B}{\hbar^{2}} V(\beta)$ are the reduced energies and potentials, respectively, while $\tau$ is the seniority quantum number [31].

In the radial part of the Hamiltonian for $u(\beta)$ the Morse potential [32] is used

$$
u(\beta)=e^{-2 a\left(\beta-\beta_{e}\right)}-2 e^{-a\left(\beta-\beta_{e}\right)},
$$

leading to the energy spectrum [16]

$$
\epsilon_{n, \tau}=\frac{\nu c_{0}}{\beta_{e}^{2}}-\left[\frac{\gamma_{1}^{2}}{2 \beta_{e} \gamma_{2}}-\left(n+\frac{1}{2}\right) \frac{\alpha}{\beta_{e}}\right]^{2},
$$


where $n$ is the principal quantum number, and

$$
\begin{array}{r}
c_{0}=1-\frac{3}{\alpha}+\frac{3}{\alpha^{2}}, \quad c_{1}=\frac{4}{\alpha}-\frac{6}{\alpha^{2}}, \quad c_{2}=-\frac{1}{\alpha}+\frac{3}{\alpha^{2}}, \\
\alpha=a \beta_{e}, \quad \gamma_{1}^{2}=2 \beta_{e}^{2}-\nu c_{1}, \quad \gamma_{2}^{2}=\beta_{e}^{2}+\nu c_{2}, \quad \nu=\tau(\tau+3)+2 .
\end{array}
$$

To get the radial wave function one needs the parametrization [16]

$$
\begin{array}{r}
\xi(\beta)=\beta^{-2} \chi(\beta), \quad x=\frac{\beta-\beta_{e}}{\beta_{e}}, \quad y=e^{-\alpha x}, \quad \chi_{n, \tau}(y)=y^{K_{n, \tau} / \alpha} e^{-\gamma_{2} y / \alpha} f_{n, \tau}(y), \\
K_{n, \tau}=\sqrt{\nu c_{0}-\epsilon_{n, \tau} \beta_{e}^{2}}
\end{array}
$$

leading to [16]

$$
f_{n, \tau}^{\prime \prime}(y)=\left(\frac{2 \gamma_{2} \alpha y-2 \alpha K_{n, \tau}-\alpha^{2}}{\alpha^{2} y}\right) f_{n, \tau}^{\prime}(y)+\left(\frac{2 K_{n, \tau} \gamma_{2}+\alpha \gamma_{2}-\gamma_{1}^{2}}{\alpha^{2} y}\right) f_{n, \tau}(y) .
$$

As described in Appendix A, the solution of this equation is found to be

$$
f_{n, \tau}(y)=N_{n, \tau} L_{n}^{2 K_{n, \tau} / \alpha}\left(\frac{2 \gamma_{2}}{\alpha} y\right)
$$

where $L$ represents the Laguerre polynomials and $N$ are normalization constants determined from the normalization condition,

$$
\int_{0}^{\infty} \xi^{2}(\beta) \beta^{4} d \beta=\int_{0}^{\infty} \chi^{2}(\beta) d \beta=\frac{1}{a} \int_{0}^{e^{\alpha}} \frac{1}{y} \chi^{2}(y) d y=1
$$

leading to

$$
N_{n, \tau}=\left[\frac{a(\omega-2 n-1) n !}{(\omega-n-1) \Gamma(\omega-n-1)}\left(\frac{2 \gamma_{2}}{\alpha}\right)^{\omega-2 n-1}\right]^{1 / 2}, \quad \omega=\frac{\gamma_{1}^{2}}{\alpha \gamma_{2}}
$$

The quadrupole operator is defined as [30]

$$
T_{\mu}^{(E 2)}=t \alpha_{\mu}=t \beta\left[D_{\mu, 0}^{(2)}\left(\theta_{i}\right) \cos \gamma+\frac{1}{\sqrt{2}}\left(D_{\mu, 2}^{(2)}\left(\theta_{i}\right)+D_{\mu,-2}^{(2)}\left(\theta_{i}\right) \sin \gamma\right)\right],
$$

where $t$ is a scale factor, while $D\left(\theta_{i}\right)$ represent the Wigner functions of the Euler angles $\theta_{i}$. Then $B(E 2)$ transition rates are given by

$$
\begin{aligned}
B\left(E 2 ; s_{i}, L_{i} \rightarrow s_{f}, L_{f}\right) & =\frac{1}{2 L_{i}+1}\left|\left\langle s_{f}, L_{f}\left\|T^{(E 2)}\right\| s_{i}, L_{i}\right\rangle\right|^{2} \\
& =\frac{2 L_{f}+1}{2 L_{i}+1} B\left(E 2 ; s_{f}, L_{f} \rightarrow s_{i}, L_{i}\right) .
\end{aligned}
$$

As mentioned in Ref. [9], the states defined in Eq. (8) with $\nu_{\Delta}=0$ (where $\nu_{\Delta}$ is the missing quantum number in the $\mathrm{SO}(5) \supset \mathrm{SO}(3)$ reduction) and $L=2 \tau$ take the form

$$
\psi\left(\beta, \gamma, \theta_{i}\right)=\xi(\beta) \phi_{\tau}\left(\gamma, \theta_{i}\right),
$$


where the functions $\phi_{\tau}\left(\gamma, \theta_{i}\right)$ are given by [33]

$$
\phi_{\tau}\left(\gamma, \theta_{i}\right)=\frac{1}{4 \pi} \sqrt{\frac{(2 \tau+3) ! !}{\tau !}}\left(\frac{\alpha_{2}}{\beta}\right)^{\tau}
$$

with $\alpha_{2}$ defined in Eq. (18). Then $B(E 2)$ s are found to be [9]

$$
\begin{aligned}
& B\left(E 2 ; L_{n, \tau} \rightarrow(L+2)_{n^{\prime}, \tau+1}\right)=\frac{(\tau+1)(4 \tau+5)}{(2 \tau+5)(4 \tau+1)} t^{2} I_{n^{\prime}, \tau+1 ; n, \tau}^{2}, \quad L=2 \tau, \\
& B\left(E 2 ;(L+2)_{n^{\prime}, \tau+1} \rightarrow L_{n, \tau}\right)=\frac{(\tau+1)}{(2 \tau+5)} t^{2} I_{n^{\prime}, \tau+1 ; n, \tau}^{2}, \quad L=2 \tau,
\end{aligned}
$$

where

$$
I_{n^{\prime}, \tau+1 ; n, \tau}=\int_{0}^{\infty} \beta \chi_{n^{\prime}, \tau+1}(\beta) \chi_{n, \tau}(\beta) d \beta .
$$

For the Morse potential, using the eigenfunctions in Eq.(13) one gets

$$
\begin{aligned}
I_{n^{\prime}, \tau+1 ; n, \tau} & =\int_{0}^{\infty} \beta^{5} \xi_{n^{\prime}, \tau+1}(\beta) \xi_{n, \tau}(\beta) d \beta \\
& =\frac{1}{a^{2}} \int_{0}^{e^{\alpha}}\left(\frac{\alpha-\ln y}{y}\right) \chi_{n^{\prime}, \tau+1}(y) \chi_{n, \tau}(y) d y .
\end{aligned}
$$

\section{B. The rotational case}

For the rotational region, the reduced potential is $\gamma$-dependent. Exact separation of the Bohr Hamiltonian can be achieved [30] by taking the reduced potential to be of the form of $u(\beta, \gamma)=u(\beta)+v(\gamma) / \beta^{2}$. Then the wave functions can be written as

$$
\psi\left(\beta, \gamma, \theta_{i}\right)=\xi_{L}(\beta) \Gamma_{K}(\gamma) D_{M, K}^{L}\left(\theta_{i}\right),
$$

where $L$ is the angular momentum quantum number, while $M$ and $K$ are the angular momentum projections on the laboratory-fixed $z$-axis and body-fixed $z^{\prime}$-axis, respectively. For an axially-symmetric prolate deformed structure, assuming $v(\gamma)=(3 c)^{2} \gamma^{2}$, the $\gamma$-part of the solution [15] is given in terms of Laguerre polynomials

$$
\Gamma_{n_{\gamma}, K}(\gamma)=N_{n_{\gamma}, K} \gamma^{|K / 2|} e^{-3 c \gamma^{2} / 2} L_{\tilde{n}}^{|K / 2|}\left(3 c \gamma^{2}\right)
$$

with

$$
n_{\gamma}=0,1,2 \ldots, \quad \tilde{n}=\frac{n_{\gamma}-|K / 2|}{2},
$$

while the normalization constants $N_{n_{\gamma}, K}$ can be found from the normalization condition

$$
\int_{0}^{\pi / 3} \Gamma_{n_{\gamma}, K}^{2}(\gamma)|\sin 3 \gamma| d \gamma=1
$$

For deformed nuclei one has $\gamma \approx 0$, which implies that one can use $|\sin 3 \gamma| \simeq 3|\gamma|$. The normalization constant for the $\left(n_{\gamma}, K\right)=(0,0)$ state is found to be

$$
N_{0,0}=\sqrt{\frac{2 c}{1-e^{-\frac{c \pi^{2}}{3}}}} .
$$


For high $c$ (say $c \geq 2$ ) the $e^{-\frac{c \pi^{2}}{3}}$ term can be neglected, since it becomes of the order of $10^{-4}$, or smaller. Then the normalization constants are as follows,

$$
\begin{array}{rll}
N_{0,0}=\sqrt{2 c} \quad, & N_{3,6}=\sqrt{9 c^{4}}, \\
N_{1,2}=\sqrt{6 c^{2}} & , & N_{4,0}=\sqrt{2 c}, \\
N_{2,0}=\sqrt{2 c} \quad, & N_{4,4}=\sqrt{3 c^{3}}, \\
N_{2,4}=\sqrt{9 c^{3}} \quad, & N_{4,8}=\sqrt{\frac{27}{4} c^{5},} \\
N_{3,2}=\sqrt{3 c^{2}} \quad, & N_{5,10}=\sqrt{\frac{81}{20} c^{6}} .
\end{array}
$$

The energy spectrum turns out to be [16]

$$
\epsilon_{n, L}=\frac{\mu c_{0}}{\beta_{e}^{2}}-\left[\frac{\gamma_{1}^{2}}{2 \beta_{e} \gamma_{2}}-\left(n+\frac{1}{2}\right) \frac{\alpha}{\beta_{e}}\right]^{2}
$$

where

$$
\begin{array}{r}
\gamma_{1}^{2}=2 \beta_{e}^{2}-\mu c_{1}, \quad \gamma_{2}^{2}=\beta_{e}^{2}+\mu c_{2}, \\
\mu=\frac{L(L+1)}{3}+2+\lambda, \quad \lambda=\epsilon_{\gamma}-\frac{K^{2}}{3}, \quad \epsilon_{\gamma}=(3 C)\left(n_{\gamma}+1\right), \quad C=2 c,
\end{array}
$$

while the rest of the symbols have the same value as in Eq. (12).

To get the radial wave function, one needs the parametrization

$$
\begin{array}{r}
\xi_{n, L}(\beta)=\beta^{-2} \chi_{n, L}(\beta), \quad x=\frac{\beta-\beta_{e}}{\beta_{e}}, \quad y=e^{-\alpha x}, \quad \chi_{n, L}(y)=y^{\rho_{n, L} / \alpha} e^{-\gamma_{2} y / \alpha} R_{n, L}(y) \\
\rho_{n, L}=\frac{\gamma_{1}^{2}}{2 \gamma_{2}}-\left(n+\frac{1}{2}\right) \alpha
\end{array}
$$

leading to

$$
R_{n, L}^{\prime \prime}(y)=\left(\frac{2 \gamma_{2} \alpha y-2 \alpha \rho_{n, L}-\alpha^{2}}{\alpha^{2} y}\right) R_{n, L}^{\prime}(y)+\left(\frac{2 \rho_{n, L} \gamma_{2}+\alpha \gamma_{2}-\gamma_{1}^{2}}{\alpha^{2} y}\right) R_{n, L}(y) .
$$

In the right-hand-side of Eq. (35), the first and the second functions in parentheses are $\lambda_{0}(y)$ and $s_{0}(y)$ of Eq. (11). After determination of $\lambda_{k}$ 's and $s_{k}$ 's, through the use of Eq. (44), the wave functions are found by using Eq. (6) to be

$$
R_{n, L}(y)=N_{n, L} L_{n}^{2 \rho_{n, L} / \alpha}\left(\frac{2 \gamma_{2}}{\alpha} y\right)
$$

where $L$ represents the Laguerre polynomials, while $N_{n, L}$ correspond to normalization constants, obtained from the normalization condition

$$
\int_{0}^{\infty} \xi^{2}(\beta) \beta^{4} d \beta=1
$$


to be

$$
N_{n, L}=\left[\frac{a(\sigma-2 n-1) n !}{(\sigma-n-1) \Gamma(\sigma-n-1)}\left(\frac{2 \gamma_{2}}{\alpha}\right)^{\sigma-2 n-1}\right]^{1 / 2}, \quad \sigma=\frac{\gamma_{1}^{2}}{\alpha \gamma_{2}} .
$$

Then one can calculate the $\mathrm{B}(\mathrm{E} 2)$ transition rates by following the procedure given in Appendix B of Ref. [15]

$$
B\left(E 2 ; n L n_{\gamma} K \rightarrow n^{\prime} L^{\prime} n_{\gamma}^{\prime} K^{\prime}\right)=\frac{5}{16 \pi} t^{2}\left(\left\langle L, 2, L^{\prime} \mid K, K^{\prime}-K, K^{\prime}\right\rangle\right)^{2} B_{n, L, n^{\prime}, L^{\prime}}^{2} C_{n_{\gamma}, K, n_{\gamma}^{\prime}, K^{\prime}}^{2}
$$

In our case, $B_{n, L, n^{\prime}, L^{\prime}}$ is given by

$$
B_{n, L, n^{\prime}, L^{\prime}}=-\frac{1}{a^{2}} \int_{e^{\alpha}}^{0} \frac{\alpha-\ln (y)}{y} \chi_{n, L}(y) \chi_{n^{\prime}, L^{\prime}}(y) d y,
$$

where $\chi_{n, L}(y)$ is defined in Eq. (34). The other constant $C_{n_{\gamma}, K, n_{\gamma}^{\prime}, K^{\prime}}$ is

$$
\begin{aligned}
C_{n_{\gamma}, K, n_{\gamma}^{\prime}, K^{\prime}} & =\int_{0}^{\pi / 3} \cos \gamma \Gamma_{n_{\gamma}^{\prime}, K^{\prime}}(\gamma) \Gamma_{n_{\gamma}, K}(\gamma)|\sin 3 \gamma| d \gamma, \quad \Delta K=0, \\
& =\int_{0}^{\pi / 3} \sin \gamma \Gamma_{n_{\gamma}^{\prime}, K^{\prime}}(\gamma) \Gamma_{n_{\gamma}, K}(\gamma)|\sin 3 \gamma| d \gamma, \quad \Delta K=2 .
\end{aligned}
$$

These integrals can be calculated by remembering that for deformed nuclei $\gamma \approx 0$, implying $\cos \gamma \simeq 1$ and $\sin \gamma \simeq \gamma$. Under these approximations, for $\Delta K=0$ transitions one has $C_{n_{\gamma}, K, n_{\gamma}^{\prime}, K^{\prime}}=\delta_{n_{\gamma}, n_{\gamma}^{\prime}} \delta_{K, K^{\prime}}$, therefore

$$
B\left(E 2 ; n L n_{\gamma} K \rightarrow n^{\prime} L^{\prime} n_{\gamma} K\right) \approx \frac{5}{16 \pi} t^{2}\left(<L 2 L^{\prime} \mid K 0 K>\right)^{2} B_{n, L, n^{\prime}, L^{\prime}}^{2}
$$

For $\Delta K=2$ transitions, if the states have $\tilde{n}=0$ [see Eq. (28)], then the Laguerre polynomials are unity [see Eq. (27)] and

$$
C_{n_{\gamma}, K, n_{\gamma}^{\prime}, K^{\prime}}=3 N_{n_{\gamma}^{\prime}, K^{\prime}} N_{n_{\gamma}, K} \int_{0}^{\pi / 3} \gamma^{\frac{4+\left|K^{\prime}\right|+|K|}{2}} e^{-3 c \gamma^{2}} d \gamma
$$

As an example, we give $C_{n_{\gamma}, K, n_{\gamma}^{\prime}, K^{\prime}}$ for the $\left(n_{\gamma}^{\prime}=1, K^{\prime}=2\right) \rightarrow\left(n_{\gamma}=0, K=0\right)$ transition,

$$
\begin{aligned}
C_{0,0,1,2} & =3 N_{0,0} N_{1,2} \int_{0}^{\pi / 3} \gamma^{3} e^{-3 c \gamma^{2}} d \gamma \\
& =3 N_{0,0} N_{1,2}\left(\frac{1}{18 c^{2}}-\left[\frac{1}{18 c^{2}}+\frac{\pi^{2}}{54 c}\right] e^{-\frac{c \pi^{2}}{3}}\right) .
\end{aligned}
$$

As explained below Eq. (30), the second term can be neglected. Then

$$
C_{0,0,1,2}=\frac{1}{6 c^{2}} N_{0,0} N_{1,2} .
$$


The $\gamma$-integrals for higher $\Delta K=2$ transitions are

$$
\begin{aligned}
C_{1,2,2,4} & =\frac{1}{9 c^{3}} N_{1,2} N_{2,4} \\
C_{2,4,3,6} & =\frac{1}{9 c^{4}} N_{2,4} N_{3,6} \\
C_{3,6,4,8} & =\frac{4}{27 c^{5}} N_{3,6} N_{4,8} \\
C_{4,8,5,10} & =\frac{20}{81 c^{6}} N_{4,8} N_{5,10} .
\end{aligned}
$$

In the above, the normalization factors $N_{n_{\gamma}, K}$ are given in Eq. (31).

\section{NUMERICAL RESULTS}

Numerical calculations for $B(E 2)$ s have been performed for $30 \gamma$-unstable nuclei, listed in the left part of Table I, as well as for 32 rotational nuclei, listed in the right part of Table [1. The parameters listed for these nuclei have been obtained in Ref. [16] by fitting the experimental energy spectra. No attempt to include $B(E 2) \mathrm{s}$ in the fit has been made. Nuclei for which at least one $B(E 2)$ value is known in addition to $B\left(E 2: 2_{1}^{+} \rightarrow 0_{1}^{+}\right.$) (to which we normalize the other $B(E 2)$ values) are included in the tables.

$B(E 2)$ s for $\gamma$-unstable nuclei are shown in Table II, while $B(E 2)$ s for rotational nuclei are shown in Table [II. It is clear from Table [I] that the Morse potential gives in general good results for the transitions within the ground state band of $\gamma$-unstable nuclei, while the predictions for some interband transitions are systematically lower than the experimental data. On the other hand, in Table III one can see that the Morse potential is successful in predicting the intraband $B(E 2)$ s within the ground state band of deformed nuclei, while most inter-band $B(E 2)$ s are systematically overpredicted.

Numerical results for the spectra and $B(E 2)$ s of the same nuclei, both in the $\gamma$-unstable and in the rotational regions, have been provided recently [35, 36] using a Bohr Hamiltonian in which the mass is not a constant, as in the present work, but it is a function of the deformation, with a Davidson potential used in the radial degree of freedom. Analytical solutions are obtained through the use of techniques of supersymmetric quantum mechanics (SUSYQM) [37]. It is instructive to compare the results of the present approach and the deformation-dependent mass (DDM) Davidson approach. The following remarks apply.

1) In both solutions the same assumptions have been made as far as the separability of the potential is concerned.

2) In both solutions the same number of free parameters (up to overall scale factors) is used both in the $\gamma$-unstable case (two parameters) and in the rotational case (three parameters).

3) The same set of experimental data has been used in both models.

4) In both models the parameters have been determined by fitting the energy levels alone. These parameters have been subsequently used for calculating the $B(E 2)$ s.

$5)$ In both models the problem of large spacings within the $\beta$-band is well accounted for.

6 ) In both models both intraband and interband $B(E 2)$ s for $\gamma$-unstable nuclei are somewhat systematically overpredicted. The intraband $B(E 2)$ s for rotational nuclei predicted by both models are in general in good agreement to the data, while some interband $B(E 2) \mathrm{s}$ are still systematically overpredicted. 
The similarity of the numerical results provided by the two models invites for a look into their differences.

1) In the DDM Davidson framework [36] it seems that the dependence of the mass on the deformation is the main factor leading to improved agreement to experiment, since the increase of the moments of inertia with deformation is moderated, as one can see by comparing the results of Ref. [36] to these of Ref. [15], where the Davidson potential with constant mass is considered. The mass dependence on the deformation is absent in the present Morse model.

2) In the present Morse model, it seems that it is the shape of the potential which leads to good agreement to experiment. It is known that the rapid increase of the potential "on the right hand side", i.e., for large $\beta$, leads to large spacings within the $\beta$ band, which are moderated if the increase of the potential is made slower [38]. The Morse potential becomes flat on the right hand side, while the Davidson potential is growing as $\beta^{2}$.

From the above it is plausible that a DDM form of the Kratzer potential [5] should be studied, since this will have both good features of the models already considered, namely the deformation dependence of the mass, used in the Davidson model, and the finiteness at large $\beta$, possessed by the Morse model. Work in this direction is in progress. It should be noticed that among the potentials soluble by SUSYQM techniques [37] with deformation dependent mass [39], only the Davidson and Kratzer potentials (i.e., harmonic oscillator and Coulomb potentials with centrifugal terms) can be successfully treated in more than one dimensions, allowing the presence of angular momentum. As of today, the Morse potential can be treated successfully by these techniques only in one dimension.

It should be mentioned that numerical solutions of the Bohr Hamiltonian avoiding the assumptions guaranteeing the separation of variables used in the present approach, can be obtained in the framework of the algebraic collective model [40-42]. Preliminary results [43] indicate that the overprediction of interband $B(E 2)$ s seems to persist even when separation of variables is avoided.

\section{CONCLUSIONS}

Using the asymptotic iteration method (AIM), the Bohr Hamiltonian with the Morse potential has been studied for $\gamma$-unstable nuclei and for deformed nuclei. Wave functions have been obtained in closed form and $B(E 2)$ transition rates have been evaluated for 30 $\gamma$-unstable and 32 rotational nuclei and compared to experimental data. Agreement to experimental data, as well as to theoretical predictions obtained with a Bohr Hamiltonian possessing a deformation-dependent mass (DDM) and using a Davidson potential is in general good for intraband transitions within the ground state band. In the case of $\gamma$-unstable nuclei, some interband transitions are systematically underpredicted by both models, while in rotational nuclei some interband $B(E 2)$ s are systematically overpredicted by both models. In order to test if this systematic overprediction is due to the shape of the potential used or to the special forms of the potentials used, which are amenable to separation of variables, a DDM calculation using a Kratzer potential is suggested.

\section{ACKNOWLEDGMENT}

This work has been partly supported by the Turkish Science and Research Council (TÜBİTAK) through Grant Numbers 109T373 and 110T388, the Turkish Academy of Sciences (TÜBA-GEBIP) and the Akdeniz University Scientific Research Projects Unit. 


\section{Appendix A: $\gamma$-unstable case}

To get the solution of the second-order differential equation of the form of Eq. (1), our starting point is Eq. (6).

Comparing Eq. (14) to Eq.(11), one can see that

$$
\begin{aligned}
& \lambda_{0}(y)=\frac{2 \gamma_{2} \alpha y-2 \alpha K_{0, \tau}-\alpha^{2}}{\alpha^{2} y}, \\
& s_{0}(y)=\frac{2 K_{0, \tau} \gamma_{2}+\alpha \gamma_{2}-\gamma_{1}^{2}}{\alpha^{2} y}
\end{aligned}
$$

where $K_{0, \tau}=\left(\gamma_{1}^{2}-\alpha \gamma_{2}\right) / 2 \gamma_{2}$ and

$$
f_{0, \tau}(y)=\left(C_{2}\right)_{0, \tau} \exp \left(-\int^{y} \frac{s_{0}}{\lambda_{0}} d y^{\prime}\right)=\left(C_{2}\right)_{0, \tau} .
$$

Then one can calculate $f_{n, \tau}$ using the definition of $\lambda_{k}$ 's and $s_{k}$ 's as given in Eq. (4), finding

$$
\begin{aligned}
\lambda_{1}= & \lambda_{0}^{\prime}(y)+s_{0}(y)+\lambda_{0}(y) \lambda_{0}(y), \quad s_{1}=s_{0}^{\prime}(y)+s_{0}(y)+\lambda_{0}(y), \quad K_{1, \tau}=\frac{\gamma_{1}^{2}-3 \alpha \gamma_{2}}{2 \gamma_{2}}, \\
f_{1, \tau}(y)= & \left(C_{2}\right)_{1, \tau} \exp \left(-\int^{y} \frac{s_{1}}{\lambda_{1}} d y^{\prime}\right)=\left(C_{2}\right)_{1, \tau}\left(2 \gamma_{2}^{2} y+2 \alpha \gamma_{2}-\gamma_{1}^{2}\right), \\
\lambda_{2}= & \lambda_{1}^{\prime}(y)+s_{1}(y)+\lambda_{0}(y) \lambda_{1}(y), \quad s_{2}=s_{1}^{\prime}(y)+s_{0}(y)+\lambda_{1}(y), \quad K_{2, \tau}=\frac{\gamma_{1}^{2}-5 \alpha \gamma_{2}}{2 \gamma_{2}}, \\
f_{2, \tau}(y)= & \left(C_{2}\right)_{2, \tau} \exp \left(-\int^{y} \frac{s_{2}}{\lambda_{2}} d y^{\prime}\right) \\
= & \left(C_{2}\right)_{2, \tau}\left(4 \gamma_{2}^{4} y^{2}+12 \gamma_{2}^{3} \alpha y-4 \gamma_{1}^{2} y \gamma_{2}^{2}+12 \alpha^{2} \gamma_{2}^{2}-7 \gamma_{1}^{2} \gamma_{2} \alpha+\gamma_{1}^{4}\right), \\
\lambda_{3}= & \lambda_{2}^{\prime}(y)+s_{2}(y)+\lambda_{0}(y) \lambda_{2}(y), \quad s_{3}=s_{2}^{\prime}(y)+s_{0}(y)+\lambda_{2}(y), \quad K_{3, \tau}=\frac{\gamma_{1}^{2}-7 \alpha \gamma_{2}}{2 \gamma_{2}}, \\
f_{3, \tau}(y)= & \left(C_{2}\right)_{3, \tau} \exp \left(-\int^{y} \frac{s_{3}}{\lambda_{3}} d y^{\prime}\right) \\
& \ldots \quad \operatorname{etc.} \quad(\mathrm{A} 5)
\end{aligned}
$$

From these expressions, it is easy to write $f_{n, \tau}(y)$ in a closed form as

$$
f_{n, \tau}(y)=\left(C_{2}\right)_{n, \tau} L_{n}^{2 K_{n, \tau} / \alpha}\left(\frac{2 \gamma_{2}}{\alpha} y\right)
$$

where $L$ represents the Laguerre polynomials. 
[1] P. Cejnar, J. Jolie, and R. F. Casten, Rev. Mod. Phys. 82, 2155 (2010).

[2] F. Iachello, Phys. Rev. Lett. 85, 3580 (2000).

[3] F. Iachello, Phys. Rev. Lett. 87, 052502 (2001).

[4] A. Bohr, Mat. Fys. Medd. K. Dan. Vidensk. Selsk. 26, no. 14 (1952).

[5] A. Kratzer, Z. Phys. 3, 289 (1920).

[6] L. Fortunato and A. Vitturi, J. Phys. G: Nucl. Part. Phys. 29, 1341 (2003).

[7] L. Fortunato and A. Vitturi, J. Phys. G: Nucl. Part. Phys. 30, 627 (2004).

[8] J. M. Arias, C. E. Alonso, A. Vitturi, J. E. García-Ramos, J. Dukelsky and A. Frank, Phys. Rev. C 68, 041302(R) (2003).

[9] D. Bonatsos, D. Lenis, N. Minkov, P. P. Raychev and P. A. Terziev, Phys. Rev. C 69, 044316 (2004).

[10] D. Bonatsos, D. Lenis, E. A. McCutchan, D. Petrellis and I. Yigitoglu, Phys. Lett. B649, 394 (2007).

[11] P. M. Davidson, Proc. R. Soc. London, Ser. A 135, 459 (1932).

[12] J. P. Elliott, J. A. Evans and P. Park, Phys. Lett. B169, 309 (1986).

[13] D. J. Rowe and C. Bahri, J. Phys. A 31, 4947 (1998).

[14] D. Bonatsos, D. Lenis, N. Minkov, D. Petrellis, P. P. Raychev and P. A. Terziev, Phys. Lett. B584, 40 (2004).

[15] D. Bonatsos, E. A. McCutchan, N. Minkov, R. F. Casten, P. Yotov, D. Lenis, D. Petrellis and I. Yigitoglu, Phys. Rev. C 76, 064312 (2007).

[16] I. Boztosun, D. Bonatsos, and I. Inci, Phys. Rev. C 77, 044302 (2008).

[17] R. F. Casten and N. V. Zamfir, Phys. Rev. Lett. 87, 052503 (2001).

[18] R. Krücken et al., Phys. Rev. Lett. 88, 232501 (2002).

[19] R. F. Casten and E. A. McCutchan, J. Phys. G: Nucl. Part. Phys. 34, R285 (2007).

[20] H. Ciftci, R. L. Hall and N. Saad, J. Phys. A: Math. Gen. 36, 11807 (2003).

[21] H. Ciftci, R. L. Hall and N. Saad, J. Phys. A: Math. Gen. 38, 1147 (2005).

[22] M. Karakoc and I. Boztosun, Int. J. Mod. Phys. E: Nucl. Phys. 15, 1253 (2006).

[23] M. Aygun, O. Bayrak and I. Boztosun, J. Phys. B: At. Mol. Opt. Phys. 40 (2007) 537.

[24] O. Bayrak and I. Boztosun, Physica Scripta 76 (2007) 92. 
[25] I. Boztosun, M. Karakoc, F. Yasuk, and A. Durmus, J. Math. Phys. 47, 062301 (2006).

[26] A. Soylu, O. Bayrak, and I. Boztosun, Int. J. Mod. Phys. E 15, 1263 (2006).

[27] O. Bayrak and I. Boztosun, J. Molec. Struct.: THEOCHEM 802, 17 (2007).

[28] O. Bayrak, G. Kocak, and I. Boztosun, J. Phys. A: Math. Gen. 39, 11521 (2006).

[29] I. Boztosun and M. Karakoc, Chin. Phys. Lett. 24, 3028 (2007).

[30] L. Wilets and M. Jean, Phys. Rev. 102, 788 (1956).

[31] G. Rakavy, Nucl. Phys. 4, 289 (1957).

[32] P. M. Morse, Phys. Rev. 34, 57 (1929).

[33] D. R. Bès, Nucl. Phys. 10, 373 (1959).

[34] Nuclear Data Sheets, as of December 2005.

[35] D. Bonatsos, P. Georgoudis, D. Lenis, N. Minkov, and C. Quesne, Phys. Lett. B 683, 264 (2010).

[36] D. Bonatsos, P. E. Georgoudis, D. Lenis, N. Minkov, and C. Quesne, Phys. Rev. C 83, 044321 (2011).

[37] F. Cooper, A. Khare, and U. Sukhatme, Supersymmetry in Quantum Mechanics (World Scientific, Singapore, 2001).

[38] M. A. Caprio, Phys. Rev. C 69, 044307 (2004).

[39] B. Bagchi, A. Banerjee, C. Quesne, and V. M. Tkachuk, J. Phys. A: Math. Gen. 38, 2929 (2005).

[40] D. J. Rowe, Nucl. Phys. A 735, 372 (2004).

[41] D. J. Rowe and P. S. Turner, Nucl. Phys. A 753, 94 (2005).

[42] D. J. Rowe, T. A. Welsh, and M. A. Caprio, Phys. Rev. C 79, 054304 (2009).

[43] M. A. Caprio, Phys. Lett. B 672, 396 (2009). 
TABLE I: Morse potential parameters for nuclei in the $\gamma$-unstable region (two columns on the left) and for nuclei in the rotational region (two columns on the right), taken from Ref. [16].

\begin{tabular}{|c|c|c|c|c|c|c|c|c|c|c|c|c|c|}
\hline nucl. & $\beta_{e}$ & $a$ & acl. & $\beta_{e}$ & $a$ & acl. & $\beta_{e}$ & $a$ & $C$ & 1. & $\beta_{e}$ & $a$ & $C$ \\
\hline $\mathrm{Ru}$ & 84 & 0.44 & $\mathrm{Xe}$ & 7.05 & 0.16 & ${ }^{4} \mathrm{Sm}$ & 8.4 & 0.20 & 13.7 & $\mathrm{rb}$ & 17.2 & 0.05 & 15.1 \\
\hline${ }^{100} \mathrm{Ru}$ & 4. & 0.36 & ${ }^{4} \mathrm{Xe}$ & 6.88 & 0.17 & ${ }^{6} \mathrm{Gd}$ & 7.5 & 0.22 & 10.5 & If & 6.1 & 0.26 & 10.4 \\
\hline${ }^{2} \mathrm{Ru}$ & 3.7 & 0.42 & ${ }^{3} \mathrm{Xe}$ & 4.74 & 0.39 & ${ }^{8} \mathrm{Gd}$ & 8.3 & 0.36 & 10.6 & $\mathrm{Hf}$ & 7.9 & 0.18 & .7 \\
\hline${ }^{104} \mathrm{Ru}$ & 7.57 & 0.10 & ${ }^{0} \mathrm{Ba}$ & 5.58 & 0.77 & ${ }^{8} \mathrm{Dy}$ & 6.7 & 0.25 & 7.1 & ${ }^{8} \mathrm{Hf}$ & 8.5 & 0.16 & 9.2 \\
\hline${ }^{102} \mathrm{Pd}$ & 4.3 & 0.34 & ${ }^{2} \mathrm{Ba}$ & 4.63 & 0.29 & ${ }^{0} \mathrm{Dy}$ & .2 & 0.18 & 8. & & 8.3 & 0.13 & 8.6 \\
\hline${ }^{104} \mathrm{Pd}$ & 4.1 & 0.41 & ${ }^{134} \mathrm{Ba}$ & 3.82 & 0.50 & ${ }^{2} \mathrm{Dy}$ & 8.3 & 0.44 & 7.2 & $\mathrm{~V}$ & 6.6 & 0.15 & 5.5 \\
\hline${ }^{106} \mathrm{Pd}$ & 3.9 & 0.43 & ${ }^{142} \mathrm{Ba}$ & 5.45 & 0.60 & ${ }^{164} \mathrm{Dy}$ & 13.1 & 0.14 & 6.8 & ${ }^{36} \mathrm{~W}$ & 5.3 & 0.22 & -2 \\
\hline${ }^{108} \mathrm{Pd}$ & 4.36 & 0.30 & ${ }^{8} \mathrm{Nd}$ & 6.40 & 0.14 & ${ }^{162} \operatorname{Er}$ & 7.1 & 0.26 & 7.0 & ${ }^{6} \mathrm{Os}$ & 5.6 & 0.27 & 4.2 \\
\hline${ }^{108} \mathrm{Cd}$ & 3.97 & 0.43 & ${ }^{152} \mathrm{Gd}$ & 3.9 & 0.40 & ${ }^{164} \mathrm{Er}$ & 9.3 & 0.1 & 6.6 & ${ }^{8} \mathrm{Os}$ & 5.3 & 0.34 & 2.8 \\
\hline${ }^{110} \mathrm{Cd}$ & 3.66 & 0.47 & ${ }^{154} \mathrm{Dy}$ & 4.2 & 0.38 & $\mathrm{Er}$ & 9.7 & 0.23 & 6. & $\mathrm{~h}$ & 7.6 & 0.1 & \\
\hline${ }^{112} \mathrm{Cd}$ & 3.5 & 0.50 & ${ }^{156} \mathrm{Er}$ & 4 & 0.34 & ${ }^{168} \mathrm{Er}$ & 1 & 0.59 & 6.7 & ${ }^{2} \mathrm{Th}$ & 9.6 & 0.1 & \\
\hline${ }^{114} \mathrm{Cd}$ & 3.43 & 0.51 & ${ }^{192} \mathrm{Pt}$ & 6.42 & 0.19 & $\mathrm{Er}$ & 6.5 & 0.17 & 9. & ${ }^{4} \mathrm{U}$ & 12. & 0. & \\
\hline${ }^{116} \mathrm{Cd}$ & 4.10 & 0.47 & ${ }^{194} \mathrm{Pt}$ & 10. & 0.14 & ${ }^{166} \mathrm{Yb}$ & 0.0 & 0.23 & 6. & ${ }^{36} \mathrm{U}$ & 13.8 & 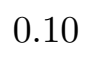 & \\
\hline${ }^{18} \mathrm{Cd}$ & 4.11 & 0.47 & ${ }^{196} \mathrm{Pt}$ & 6.26 & 0.15 & ${ }^{8} \mathrm{Yb}$ & 7.9 & 0.19 & 8.0 & ${ }^{38} \mathrm{U}$ & 13.9 & 0. & \\
\hline \multirow[t]{2}{*}{${ }^{118} \mathrm{Xe}$} & 5.40 & 0.19 & ${ }^{198} \mathrm{Pt}$ & 3.87 & 0.39 & ${ }^{170} \mathrm{Yb}$ & 7.8 & 0.49 & 9.2 & ${ }^{238} \mathrm{Pu}$ & 9.8 & 0.50 & 1 \\
\hline & & & & & & ${ }^{172} \mathrm{Yb}$ & 7.8 & 0.17 & 13 & ${ }^{250} \mathrm{Cf}$ & 12.8 & 0.20 & \\
\hline
\end{tabular}


TABLE II: Comparison of experimental data 34] (upper line) for several $B(E 2)$ ratios of $\gamma$-unstable nuclei to predictions (lower line) by the Bohr Hamiltonian with the Morse potential, for the pa-

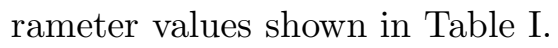

\begin{tabular}{|c|c|c|c|c|c|c|c|c|}
\hline \multirow[t]{2}{*}{ nucl. } & \multirow[t]{2}{*}{$\frac{4_{1} \rightarrow 2_{1}}{2_{1} \rightarrow 0_{1}}$} & \multirow[t]{2}{*}{$\frac{6_{1} \rightarrow 4_{1}}{2_{1} \rightarrow 0_{1}}$} & \multirow[t]{2}{*}{$\frac{8_{1} \rightarrow 6_{1}}{2_{1} \rightarrow 0_{1}}$} & \multirow[t]{2}{*}{$\frac{10_{1} \rightarrow 8_{1}}{2_{1} \rightarrow 0_{1}}$} & \multirow[t]{2}{*}{$\frac{2_{2} \rightarrow 2_{1}}{2_{1} \rightarrow 0_{1}}$} & $\frac{2_{2} \rightarrow 0_{1}}{2_{1} \rightarrow 0_{1}}$ & \multirow[t]{2}{*}{$\frac{0_{2} \rightarrow 2_{1}}{2_{1} \rightarrow 0_{1}}$} & \multirow{2}{*}{$\begin{array}{l}\frac{2_{3} \rightarrow 0_{1}}{2_{1} \rightarrow 0_{1}} \\
\times 10^{3} \\
\end{array}$} \\
\hline & & & & & & $\mathrm{x} 10^{3}$ & & \\
\hline \multirow[t]{2}{*}{${ }^{98} \mathrm{Ru}$} & $1.44(25)$ & & & & $1.62(61)$ & $36.0(152)$ & & \\
\hline & 1.71 & 2.52 & 3.61 & 5.27 & 1.71 & 0.0 & 0.82 & 4.24 \\
\hline \multirow[t]{2}{*}{${ }^{100} \mathrm{Ru}$} & $1.45(13)$ & & & & $0.64(12)$ & $41.1(52)$ & $0.98(15)$ & \\
\hline & 1.65 & 2.31 & 3.07 & 4.00 & 1.65 & 0.0 & 0.72 & 8.20 \\
\hline \multirow[t]{2}{*}{${ }^{102} \mathrm{Ru}$} & $1.50(24)$ & & & & $0.62(7)$ & $24.8(7)$ & $0.80(14)$ & \\
\hline & 1.73 & 2.53 & 3.54 & 4.86 & 1.73 & 0.0 & 0.88 & 4.61 \\
\hline \multirow[t]{2}{*}{${ }^{104} \mathrm{Ru}$} & $1.18(28)$ & & & & $0.63(15)$ & $35.0(84)$ & $0.42(7)$ & \\
\hline & 1.62 & 2.10 & 2.49 & 2.80 & 1.62 & 0.0 & 0.71 & 16.34 \\
\hline \multirow[t]{2}{*}{${ }^{102} \mathrm{Pd}$} & $1.56(19)$ & & & & $0.46(9)$ & $128.8(735)$ & & \\
\hline & 1.68 & 2.35 & 3.07 & 3.86 & 1.68 & 0.0 & 0.80 & 8.20 \\
\hline \multirow[t]{2}{*}{${ }^{104} \mathrm{Pd}$} & $1.36(27)$ & & & & $0.61(8)$ & $33.3(74)$ & & \\
\hline & 1.67 & 2.39 & 3.30 & 4.60 & 1.67 & 0.0 & 0.73 & 6.18 \\
\hline \multirow[t]{2}{*}{${ }^{106} \mathrm{Pd}$} & $1.63(28)$ & & & & $0.98(12)$ & $26.2(31)$ & $0.67(18)$ & \\
\hline & 1.70 & 2.47 & 3.51 & 5.03 & 1.70 & 0.0 & 0.79 & 4.84 \\
\hline \multirow[t]{2}{*}{${ }^{108} \mathrm{Pd}$} & $1.47(20)$ & $2.16(28)$ & $2.99(48)$ & & $1.43(14)$ & $16.6(18)$ & $1.05(13)$ & $1.90(29)$ \\
\hline & 1.68 & 2.32 & 2.95 & 3.53 & 1.68 & 0.0 & 0.85 & 9.45 \\
\hline \multirow[t]{2}{*}{${ }^{108} \mathrm{Cd}$} & $1.54(24)$ & & & & $0.64(20)$ & $67.7(120)$ & & \\
\hline & 1.69 & 2.46 & 3.48 & 5.00 & 1.69 & 0.0 & 0.77 & 4.99 \\
\hline \multirow[t]{2}{*}{${ }^{110} \mathrm{Cd}$} & $1.68(24)$ & & & & $1.09(19)$ & $48.9(78)$ & & $9.85(595)$ \\
\hline & 1.74 & 2.62 & 3.90 & 6.05 & 1.74 & 0.0 & 0.84 & 2.76 \\
\hline \multirow[t]{2}{*}{${ }^{112} \mathrm{Cd}$} & $2.02(22)$ & & & & $0.50(10)$ & $19.9(35)$ & $1.69(48)$ & $11.26(210)$ \\
\hline & 1.76 & 2.70 & 4.20 & 4.97 & 1.76 & 0.0 & 0.81 & 1.56 \\
\hline \multirow[t]{2}{*}{${ }^{114} \mathrm{Cd}$} & $1.99(25)$ & $3.83(72)$ & $2.73(97)$ & & $0.71(24)$ & $15.4(29)$ & $0.88(11)$ & $10.61(193)$ \\
\hline & 1.78 & 2.79 & 4.42 & 3.44 & 1.78 & 0.0 & 0.85 & 0.99 \\
\hline
\end{tabular}


TABLE II: (continued)

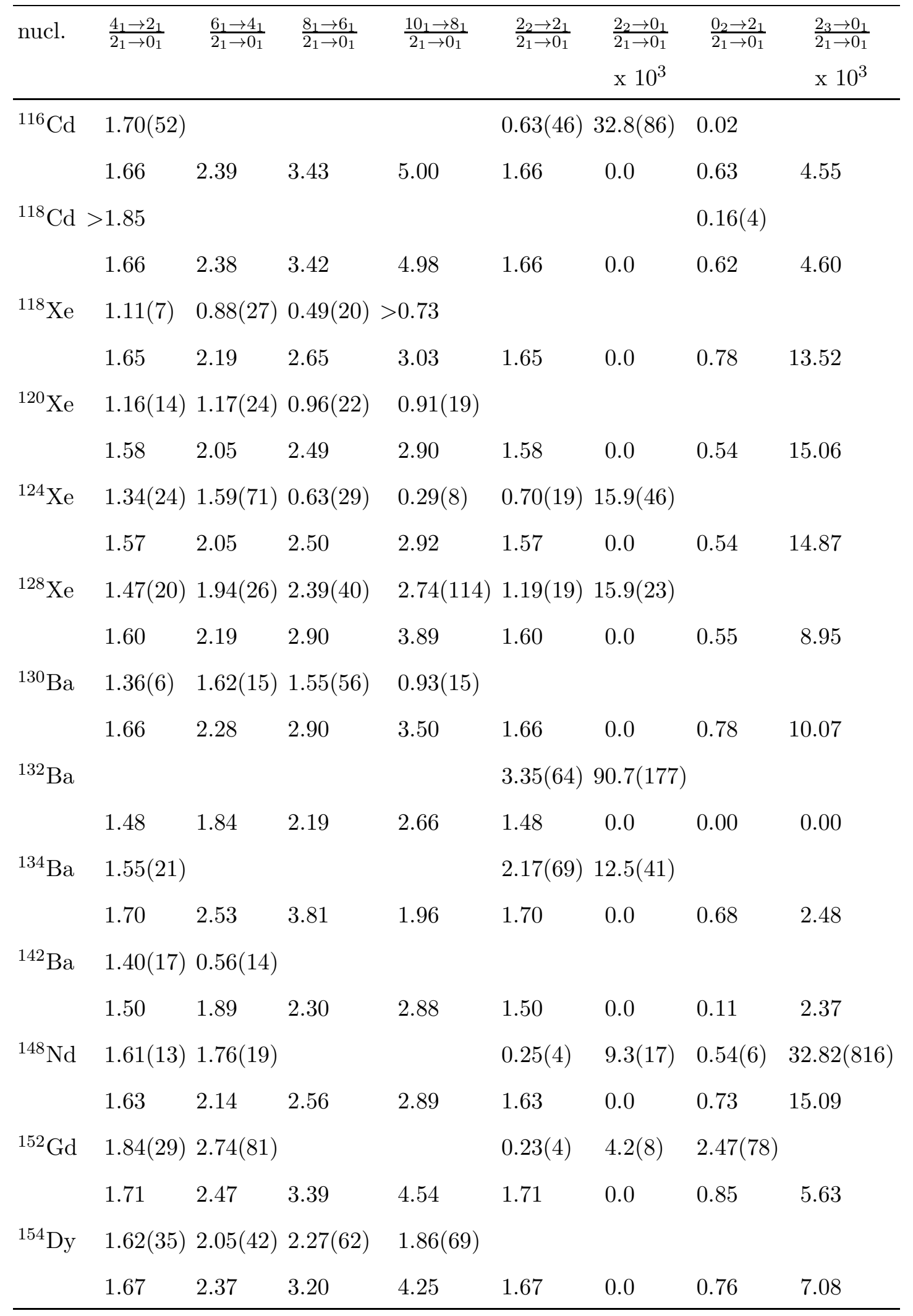


TABLE II: (continued)

\begin{tabular}{|c|c|c|c|c|c|c|c|c|}
\hline \multirow[t]{2}{*}{ nucl. } & \multirow[t]{2}{*}{$\frac{4_{1} \rightarrow 2_{1}}{2_{1} \rightarrow 0_{1}}$} & \multirow[t]{2}{*}{$\frac{6_{1} \rightarrow 4_{1}}{2_{1} \rightarrow 0_{1}}$} & \multirow[t]{2}{*}{$\frac{8_{1} \rightarrow 6_{1}}{2_{1} \rightarrow 0_{1}}$} & \multirow[t]{2}{*}{$\frac{10_{1} \rightarrow 8_{1}}{2_{1} \rightarrow 0_{1}}$} & \multirow[t]{2}{*}{$\frac{2_{2} \rightarrow 2_{1}}{2_{1} \rightarrow 0_{1}}$} & $\frac{2_{2} \rightarrow 0_{1}}{2_{1} \rightarrow 0_{1}}$ & \multirow[t]{2}{*}{$\frac{0_{2} \rightarrow 2_{1}}{2_{1} \rightarrow 0_{1}}$} & \multirow{2}{*}{$\begin{array}{l}\frac{2_{3} \rightarrow 0_{1}}{2_{1} \rightarrow 0_{1}} \\
\mathrm{x} 10^{3}\end{array}$} \\
\hline & & & & & & $\times 10^{3}$ & & \\
\hline \multirow[t]{2}{*}{${ }^{156} \mathrm{Er}$} & $1.78(16)$ & $1.89(36)$ & $0.76(20)$ & $0.88(22)$ & & & & \\
\hline & 1.62 & 2.23 & 2.91 & 3.74 & 1.62 & 0.0 & 0.64 & 9.60 \\
\hline \multirow[t]{2}{*}{${ }^{192} \mathrm{Pt}$} & $1.56(12)$ & $1.23(55)$ & & & $1.91(16)$ & $9.5(9)$ & & \\
\hline & 1.58 & 2.08 & 2.55 & 3.00 & 1.58 & 0.0 & 0.56 & 14.25 \\
\hline \multirow[t]{2}{*}{${ }^{194} \mathrm{Pt}$} & $1.73(13)$ & $1.36(45)$ & $1.02(30)$ & $0.69(19)$ & $1.81(25)$ & $5.9(9)$ & 0.01 & \\
\hline & 1.58 & 2.06 & 2.49 & 2.88 & 1.58 & 0.0 & 0.58 & 15.34 \\
\hline \multirow[t]{2}{*}{${ }^{196} \mathrm{Pt}$} & $1.48(3)$ & $1.80(23)$ & $1.92(23)$ & & & 0.4 & $0.07(4)$ & $0.06(6)$ \\
\hline & 1.63 & 2.14 & 2.57 & 2.93 & 1.63 & 0.0 & 0.72 & 14.74 \\
\hline \multirow[t]{2}{*}{${ }^{198} \mathrm{Pt}$} & $1.19(13)$ & $>1.78$ & & & $1.16(23)$ & $1.2(4)$ & $0.81(22)$ & $1.56(126)$ \\
\hline & 1.72 & 2.48 & 3.35 & 4.36 & 1.72 & 0.0 & 0.89 & 5.78 \\
\hline
\end{tabular}


TABLE III: Comparison of experimental data [34] (upper line) for several $B(E 2)$ ratios of axially symmetric prolate deformed nuclei to predictions (lower line) by the Bohr Hamiltonian with the Morse potential, for the parameter values shown in Table I

\begin{tabular}{|c|c|c|c|c|c|c|c|c|c|c|}
\hline \multirow[t]{2}{*}{ nucl. } & \multirow[t]{2}{*}{$\frac{4_{1} \rightarrow 2_{1}}{2_{1} \rightarrow 0_{1}}$} & \multirow[t]{2}{*}{$\frac{6_{1} \rightarrow 4_{1}}{2_{1} \rightarrow 0_{1}}$} & \multirow[t]{2}{*}{$\frac{8_{1} \rightarrow 6_{1}}{2_{1} \rightarrow 0_{1}}$} & \multirow[t]{2}{*}{$\frac{10_{1} \rightarrow 8_{1}}{2_{1} \rightarrow 0_{1}}$} & \multirow{2}{*}{$\begin{array}{l}\frac{22_{\beta} \rightarrow 0_{1}}{2_{1} \rightarrow 0_{1}} \\
\times 10^{3}\end{array}$} & \multirow{2}{*}{$\begin{array}{l}\frac{2_{\beta} \rightarrow 2_{1}}{2_{1} \rightarrow 0_{1}} \\
\mathrm{x} 10^{3}\end{array}$} & \multirow{2}{*}{$\begin{array}{l}\frac{2_{\beta} \rightarrow 4_{1}}{2_{1} \rightarrow 0_{1}} \\
\times 10^{3}\end{array}$} & \multirow{2}{*}{$\begin{array}{l}\frac{2_{\gamma} \rightarrow 0_{1}}{2_{1} \rightarrow 0_{1}} \\
\times 10^{3}\end{array}$} & \multirow{2}{*}{$\begin{array}{l}\frac{2 \gamma \rightarrow 2_{1}}{2_{1} \rightarrow 0_{1}} \\
\times 10^{3}\end{array}$} & \multirow{2}{*}{$\begin{array}{l}\frac{2 \gamma \rightarrow 4_{1}}{2_{1} \rightarrow 0_{1}} \\
\times 10^{3}\end{array}$} \\
\hline & & & & & & & & & & \\
\hline \multirow[t]{2}{*}{${ }^{154} \mathrm{Sm}$} & $1.40(5)$ & $1.67(7)$ & $1.83(11)$ & $1.81(11)$ & $5.4(13)$ & & $25(6)$ & $18.4(34)$ & & $3.9(7)$ \\
\hline & 1.46 & 1.67 & 1.85 & 2.03 & 21.1 & 39.8 & 122 & 47.3 & 69.2 & 3.6 \\
\hline \multirow[t]{2}{*}{${ }^{156} \mathrm{Gd}$} & $1.41(5)$ & $1.58(6)$ & $1.71(10)$ & $1.68(9)$ & $3.4(3)$ & $18(2)$ & $22(2)$ & $25.0(15)$ & $38.7(24)$ & $4.1(3)$ \\
\hline & 1.47 & 1.70 & 1.90 & 2.12 & 22.5 & 44.3 & 145 & 63.0 & 92.4 & 4.9 \\
\hline \multirow[t]{2}{*}{${ }^{158} \mathrm{Gd}$} & $1.46(5)$ & & $1.67(16)$ & $1.72(16)$ & $1.6(2)$ & $0.4(1)$ & $7.0(8)$ & $17.2(20)$ & $30.3(45)$ & $1.4(2)$ \\
\hline & 1.45 & 1.64 & 1.79 & 1.95 & 14.6 & 26.7 & 79 & 63.3 & 91.7 & 4.7 \\
\hline \multirow[t]{2}{*}{${ }^{158} \mathrm{Dy}$} & $1.45(10)$ & $1.86(12)$ & $1.86(38)$ & $1.75(28)$ & $12(3)$ & $19(4)$ & $66(16)$ & $32.2(78)$ & $103.8(258)$ & $11.5(48)$ \\
\hline & 1.48 & 1.73 & 1.98 & 2.26 & 23.5 & 49.0 & 175 & 95.1 & 140.1 & 7.5 \\
\hline \multirow[t]{2}{*}{${ }^{160} \mathrm{Dy}$} & $1.46(7)$ & $1.23(7)$ & $1.70(16)$ & $1.69(9)$ & $3.4(4)$ & & $8.5(10)$ & $23.2(21)$ & $43.8(42)$ & $3.1(3)$ \\
\hline & 1.46 & 1.67 & 1.84 & 2.02 & 20.9 & 39.3 & 120 & 82.7 & 120.1 & 6.2 \\
\hline \multirow[t]{2}{*}{${ }^{162} \mathrm{Dy}$} & $1.45(7)$ & $1.51(10)$ & $1.74(10)$ & $1.76(13)$ & & & & $0.12(1)$ & 0.20 & 0.02 \\
\hline & 1.45 & 1.62 & 1.76 & 1.88 & 11.4 & 20.1 & 56 & 94.3 & 135.6 & 6.9 \\
\hline \multirow[t]{2}{*}{${ }^{164} \mathrm{Dy}$} & $1.30(7)$ & $1.56(7)$ & $1.48(9)$ & $1.69(9)$ & & & & $19.1(22)$ & $38.3(39)$ & $4.6(5)$ \\
\hline & 1.44 & 1.62 & 1.74 & 1.85 & 16.1 & 27.6 & 72 & 99.7 & 143.2 & 7.3 \\
\hline \multirow[t]{2}{*}{${ }^{162} \mathrm{Er}$} & & & & & $8(7)$ & & $170(90)$ & $32.5(28)$ & $77.0(56)$ & $9.4(69)$ \\
\hline & 1.47 & 1.72 & 1.95 & 2.21 & 22.0 & 45.1 & 158 & 96.6 & 141.7 & 7.5 \\
\hline \multirow[t]{2}{*}{${ }^{164} \mathrm{Er}$} & $1.18(13)$ & & $1.57(9)$ & $1.64(11)$ & & & & $23.9(35)$ & $52.3(72)$ & $7.8(12)$ \\
\hline & 1.47 & 1.69 & 1.87 & 2.05 & 23.4 & 44.8 & 139 & 103.3 & 150.3 & 7.8 \\
\hline \multirow[t]{2}{*}{${ }^{166} \mathrm{Er}$} & $1.45(12)$ & $1.62(22)$ & $1.71(25)$ & $1.73(23)$ & & & & $25.7(31)$ & $45.3(54)$ & $3.1(4)$ \\
\hline & 1.45 & 1.64 & 1.78 & 1.92 & 17.3 & 30.9 & 88 & 104.5 & 150.7 & 7.7 \\
\hline \multirow[t]{2}{*}{${ }^{168} \mathrm{Er}$} & $1.54(7)$ & $2.13(16)$ & $1.69(11)$ & $1.46(11)$ & & & & $23.2(15)$ & $41.1(31)$ & $3.0(3)$ \\
\hline & 1.44 & 1.61 & 1.73 & 1.84 & 1.8 & 3.1 & 8 & 101.1 & 145.1 & 7.3 \\
\hline \multirow[t]{2}{*}{${ }^{170} \mathrm{Er}$} & & & $1.78(15)$ & $1.54(11)$ & $1.4(1)$ & $0.2(2)$ & $6.8(12)$ & $17.7(9)$ & & $1.4(4)$ \\
\hline & 1.46 & 1.68 & 1.84 & 1.98 & 28.1 & 50.6 & 139 & 76.1 & 110.6 & 5.7 \\
\hline
\end{tabular}


TABLE III: (continued)

\begin{tabular}{|c|c|c|c|c|c|c|c|c|c|c|}
\hline nucl. & $\frac{4_{1} \rightarrow 2_{1}}{2_{1} \rightarrow 0_{1}}$ & $\frac{6_{1} \rightarrow 4_{1}}{2_{1} \rightarrow 0_{1}}$ & $\frac{8_{1} \rightarrow 6_{1}}{2_{1} \rightarrow 0_{1}}$ & $\frac{10_{1} \rightarrow 8_{1}}{2_{1} \rightarrow 0_{1}}$ & $\begin{array}{l}\frac{2_{\beta} \rightarrow 0_{1}}{2_{1} \rightarrow 0_{1}} \\
\times 10^{3}\end{array}$ & $\begin{array}{l}\frac{2_{\beta} \rightarrow 2_{1}}{2_{1} \rightarrow 0_{1}} \\
\times 10^{3}\end{array}$ & $\begin{array}{l}\frac{2_{\beta} \rightarrow 4_{1}}{2_{1} \rightarrow 0_{1}} \\
\times 10^{3}\end{array}$ & $\begin{array}{l}\frac{2_{\gamma} \rightarrow 0_{1}}{2_{1} \rightarrow 0_{1}} \\
\times 10^{3}\end{array}$ & $\begin{array}{l}\frac{2_{\gamma} \rightarrow 2_{1}}{2_{1} \rightarrow 0_{1}} \\
\times 10^{3}\end{array}$ & $\begin{array}{l}\frac{2_{\gamma} \rightarrow 4_{1}}{2} 2_{1} \rightarrow 0_{1} \\
\times 10^{3}\end{array}$ \\
\hline${ }^{166} \mathrm{Yb}$ & $1.43(9)$ & $1.53(10)$ & $1.70(18)$ & $1.61(80)$ & & & & & & \\
\hline & 1.48 & 1.73 & 1.97 & 2.23 & 24.3 & 50.3 & 176 & 101.4 & 149.0 & 7.9 \\
\hline${ }^{168} \mathrm{Yb}$ & & & & & $8.6(9)$ & & & $22.0(55)$ & $45.9(73)$ & 8.6 \\
\hline & 1.47 & 1.70 & 1.90 & 2.11 & 23.3 & 45.7 & 148 & 84.6 & 123.7 & 6.5 \\
\hline${ }^{170} \mathrm{Yb}$ & & & $1.79(16)$ & $1.77(14)$ & $5.4(10)$ & & & $13.4(34)$ & $23.9(57)$ & $2.4(6)$ \\
\hline & 1.44 & 1.64 & 1.78 & 1.93 & 8.3 & 15.1 & 44 & 73.6 & 106.3 & 5.4 \\
\hline${ }^{172} \mathrm{Yb}$ & $1.42(10)$ & $1.51(14)$ & $1.89(19)$ & $1.77(11)$ & $1.1(1)$ & $3.7(6)$ & $12(1)$ & $6.3(6)$ & & $0.6(1)$ \\
\hline & 1.46 & 1.67 & 1.83 & 1.99 & 23.6 & 43.1 & 124 & 48.7 & 70.9 & 3.7 \\
\hline${ }^{174} \mathrm{Yb}$ & $1.39(7)$ & $1.84(26)$ & $1.93(12)$ & $1.67(12)$ & & & & & $12.4(29)$ & \\
\hline & 1.45 & 1.63 & 1.75 & 1.86 & 18.9 & 32.3 & 88 & 44.6 & 64.3 & 3.3 \\
\hline${ }^{176} \mathrm{Yb}$ & $1.49(15)$ & $1.63(14)$ & $1.65(28)$ & $1.76(18)$ & & & & 9.8 & & \\
\hline & 1.44 & 1.62 & 1.74 & 1.87 & 0.5 & 1.0 & 3 & 65.1 & 93.7 & 4.8 \\
\hline${ }^{174} \mathrm{Hf}$ & & & & & $14(4)$ & & $9(3)$ & $31.6(161)$ & $48.7(124)$ & \\
\hline & 1.46 & 1.66 & 1.81 & 1.97 & 20.3 & 37.3 & 109 & 65.4 & 94.9 & 4.9 \\
\hline${ }^{176} \mathrm{Hf}$ & & & & & $5.4(11)$ & & $31(6)$ & $21.3(26)$ & & \\
\hline & 1.46 & 1.68 & 1.86 & 2.04 & 23.3 & 43.9 & 133 & 57.0 & 83.2 & 4.4 \\
\hline${ }^{178} \mathrm{Hf}$ & & $1.38(9)$ & $1.49(6)$ & $1.62(7)$ & $0.4(2)$ & & $2.4(9)$ & $24.5(39)$ & $27.7(28)$ & $1.6(2)$ \\
\hline & 1.46 & 1.68 & 1.86 & 2.04 & 23.3 & 44.3 & 136 & 73.5 & 107.2 & 5.6 \\
\hline${ }^{182} \mathrm{~W}$ & $1.43(8)$ & $1.46(16)$ & $1.53(14)$ & $1.48(14)$ & $6.6(6)$ & $4.6(6)$ & $13(1)$ & $24.8(12)$ & $49.2(24)$ & 0.2 \\
\hline & 1.46 & 1.68 & 1.85 & 2.00 & 25.7 & 47.4 & 137 & 79.3 & 115.4 & 6.0 \\
\hline${ }^{184} \mathrm{~W}$ & $1.35(12)$ & $1.54(9)$ & $2.00(18)$ & $2.45(51)$ & $1.8(3)$ & & $24(3)$ & $37.1(28)$ & $70.6(51)$ & $4.0(4)$ \\
\hline & 1.48 & 1.71 & 1.88 & 2.04 & 30.5 & 57.4 & 167 & 124.8 & 182.0 & 9.4 \\
\hline${ }^{186} \mathrm{~W}$ & $1.30(9)$ & $1.69(12)$ & $1.60(12)$ & $1.36(36)$ & & & & $41.7(92)$ & $91.0(201)$ & \\
\hline & 1.49 & 1.76 & 1.99 & 2.20 & 31.4 & 64.7 & 213 & 164.0 & 241.2 & 12.7 \\
\hline${ }^{186} \mathrm{Os}$ & $1.45(7)$ & $1.99(7)$ & $1.89(11)$ & $2.06(44)$ & & & & $109.4(71)$ & $254.6(150)$ & $13.0(47)$ \\
\hline & 1.50 & 1.80 & 2.11 & 2.45 & 26.2 & 59.5 & 235 & 163.5 & 242.2 & 13.0 \\
\hline
\end{tabular}


TABLE III: (continued)

\begin{tabular}{|c|c|c|c|c|c|c|c|c|c|c|}
\hline \multirow[t]{2}{*}{ nucl. } & \multirow[t]{2}{*}{$\frac{4_{1} \rightarrow 2_{1}}{2_{1} \rightarrow 0_{1}}$} & \multirow[t]{2}{*}{$\frac{6_{1} \rightarrow 4_{1}}{2_{1} \rightarrow 0_{1}}$} & \multirow[t]{2}{*}{$\frac{8_{1} \rightarrow 6_{1}}{2_{1} \rightarrow 0_{1}}$} & \multirow[t]{2}{*}{$\frac{10_{1} \rightarrow 8_{1}}{2_{1} \rightarrow 0_{1}}$} & \multirow{2}{*}{$\begin{array}{l}\frac{2_{\beta} \rightarrow 0_{1}}{2_{1} \rightarrow 0_{1}} \\
\times 10^{3} \\
\end{array}$} & \multirow{2}{*}{$\begin{array}{l}\frac{2_{\beta} \rightarrow 2_{1}}{2_{1} \rightarrow 0_{1}} \\
\mathrm{x} 10^{3} \\
\end{array}$} & \multirow{2}{*}{$\begin{array}{l}\frac{2_{\beta} \rightarrow 4_{1}}{2_{1} \rightarrow 0_{1}} \\
\times 10^{3} \\
\end{array}$} & \multirow{2}{*}{$\begin{array}{l}\frac{2_{\gamma} \rightarrow 0_{1}}{2_{1} \rightarrow 0_{1}} \\
\times 10^{3}\end{array}$} & \multirow{2}{*}{$\begin{array}{l}\frac{2_{\gamma} \rightarrow 2_{1}}{2_{1} \rightarrow 0_{1}} \\
\times 10^{3}\end{array}$} & \multirow{2}{*}{$\begin{array}{l}\frac{2_{\gamma} \rightarrow 4_{1}}{2_{1} \rightarrow 0_{1}} \\
\times 10^{3}\end{array}$} \\
\hline & & & & & & & & & & \\
\hline \multirow[t]{2}{*}{${ }^{188} \mathrm{Os}$} & $1.68(11)$ & $1.75(11)$ & $2.04(15)$ & $2.38(32)$ & & & & $63.3(92)$ & $202.5(304)$ & $43.0(74)$ \\
\hline & 1.51 & 1.84 & 2.22 & 2.73 & 23.0 & 56.7 & 257 & 245.3 & 363.5 & 19.4 \\
\hline \multirow[t]{2}{*}{${ }^{230} \mathrm{Th}$} & $1.36(8)$ & & & & $5.7(26)$ & & $20(11)$ & $15.6(59)$ & $28.1(100)$ & $1.8(11)$ \\
\hline & 1.47 & 1.69 & 1.88 & 2.07 & 23.6 & 45.3 & 141 & 62.5 & 91.4 & 4.8 \\
\hline \multirow[t]{2}{*}{${ }^{232} \mathrm{Th}$} & $1.44(15)$ & $1.65(14)$ & $1.73(12)$ & $1.82(15)$ & $14(6)$ & $2.6(13)$ & $17(8)$ & $14.6(28)$ & $36.4(56)$ & 0.7 \\
\hline & 1.46 & 1.66 & 1.82 & 1.98 & 21.3 & 39.1 & 115 & 56.7 & 82.4 & 4.3 \\
\hline \multirow[t]{2}{*}{${ }^{234} \mathrm{U}$} & & & & & & & & $12.5(27)$ & $21.1(44)$ & $1.2(3)$ \\
\hline & 1.45 & 1.63 & 1.77 & 1.89 & 18.5 & 32.4 & 88 & 42.8 & 62.0 & 3.2 \\
\hline \multirow[t]{2}{*}{${ }^{236} \mathrm{U}$} & $1.42(11)$ & $1.55(11)$ & $1.59(17)$ & $1.46(17)$ & & & & & & \\
\hline & 1.45 & 1.63 & 1.75 & 1.87 & 17.6 & 30.4 & 80 & 44.6 & 64.4 & 3.3 \\
\hline \multirow[t]{2}{*}{${ }^{238} \mathrm{U}$} & & & $1.45(23)$ & $1.71(22)$ & $1.4(6)$ & $3.6(14)$ & $12(5)$ & $10.8(8)$ & $18.9(17)$ & $1.2(1)$ \\
\hline & 1.45 & 1.62 & 1.75 & 1.86 & 17.2 & 29.6 & 77 & 37.7 & 54.4 & 2.8 \\
\hline \multirow[t]{2}{*}{${ }^{238} \mathrm{Pu}$} & & & & & $14(4)$ & & $11(4)$ & & & \\
\hline & 1.44 & 1.60 & 1.70 & 1.78 & 6.1 & 10.0 & 24 & 42.4 & 60.8 & 3.1 \\
\hline \multirow[t]{2}{*}{${ }^{250} \mathrm{Cf}$} & & & & & & & & $6.8(17)$ & $10.9(25)$ & $0.6(1)$ \\
\hline & 1.44 & 1.60 & 1.71 & 1.80 & 13.0 & 21.6 & 53 & 40.1 & 57.7 & 2.9 \\
\hline
\end{tabular}

